\begin{tabular}{|c|c|}
\hline \multirow{3}{*}{ 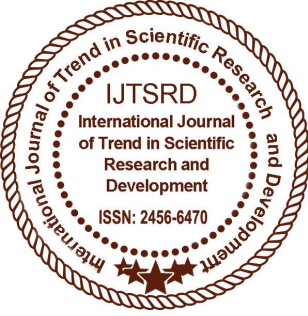 } & $\begin{array}{l}\text { International Journal of Trend in Scientific } \\
\text { Research and Development (IJTSRD) }\end{array}$ \\
\hline & International Open Access Journal \\
\hline & ISSN No: 2456 - 6470 | www.ijtsrd.com | Volume - 2 | Issue - 4 \\
\hline
\end{tabular}

\title{
Review Paper on MIMO-OFDM System Using Wimax Model
}

\author{
Anokchand Rathod, Megha Gupta \\ Department of Electronics and Communication, Lakshmi Narain College of Technology \& Science, \\ Indore, Madhya Pradesh, India
}

\begin{abstract}
Nowadays, thanks to the remarkable progress in wireless technology, affordable wireless communication service has become a reality. Wireless communication systems can be found all around the world today. WiMAX which represents (Worldwide Interoperability for Microwave Access) is a major part of broad band wireless network having IEEE 802.16 standard provides innovative fixed as well as mobile platform for broad-band internet access anywhere in anytime. IEEE 802.16 standard has bandwidth of $2 \mathrm{GHz}-11 \mathrm{GHz}$ for fixed applications and $2-6 \mathrm{GHz}$ for mobile applications. It is considered the most interesting opportunity which is able to provide data throughput up to $70 \mathrm{Mbps}$ and radio coverage distances of almost 50 kilometres, and to complete wired network architectures, ensuring a cheap flexible solution for the last-mile. WiMAX can be seen as the fourth generation $(4 \mathrm{G})$ of mobile communications systems, In this paper study on MIMO-OFDM System and review different paper on the based of WiMAX technology.
\end{abstract}

Keywords: WiMAX, MIMO, OFDM, BER, SNR

\section{INTRODUCTION}

Cellular phone systems, WLAN, wide-area wireless data systems, ad-hoc wireless networks and satellite systems etc are wireless communication. All emerged based on wireless technology to provide higher throughput, immense mobility, longer range, robust backbone to thereat. The vision extended a bit more by the engineers to provide smooth transmission of multimedia anywhere on the globe through variety of applications and devices leading a new concept of wireless communication which is cheap and flexible to implement even in odd environment.

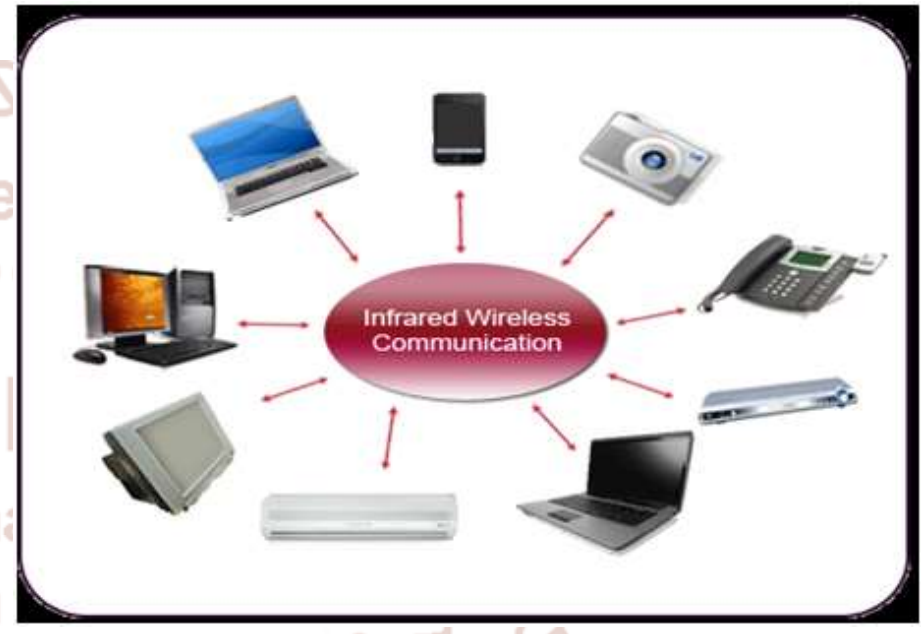

Fig. 1: Working of Infrared Wireless Communication

\section{A. WiMAX/Metropolitan Area Networks (MAN)}

"Wireless" on its own is typically used to refer to products and services from the cellular telecommunications industry. CTIA, "the Wireless Association", for example, is comprised of wireless carriers (e.g. Verizon, AT\&T, T-Mobile, and Sprint), cell phones manufacturers like Motorola and Samsung and others in the mobile phone market. Different wireless (cellular) protocols and phone standards include CDMA, GSM, EV-DO, 3G, and 4G. The term "wireless internet" most often refers to cellular data, though the phrase can also mean data access via satellite. WiMAX is a wireless digital communications system, also known as IEEE 802.16 that is intended for wireless "metropolitan area networks". WiMAX can provide broadband wireless access up to 30 miles $(50 \mathrm{~km})$ for fixed stations, and 3 - 10 miles $(5-15 \mathrm{~km})$ for mobile stations. It is maintained by the WiMAX Forum industry alliance. 
The Forum promotes and certifies compatibility and interoperability of products based on the IEEE 802.16 standards [2]. With the rapid increasing in the demand of the communications, the existing simple data communications is being changed to the multimedia data communications served in the image, audio, video and various styles. Specially, the multimedia communication services are based on the large amount of the transmission data and the reliable transmission, so the focus of the efficient management of the communication is concentrated [3].

\section{LITERATURE SURVEY}

1. Rohtang Saxena \& Neeraj Shrivastava (2016). “Energy Efficient System Using MIMO-OFDM" International Journal of Electrical, Electronics And Data Communication, Volume-4, Issue-12. In this paper we demonstrate a method by using Alamouti Codes for OFDM system with Linear MMSE detection techniques. The orthogonal principle introduced in OFDM helps to avoid Inter Symbol Interference while Alamouti provides signal security with reducing errors especially at high Sound to Noise ratio regime. We have introduced White Gaussian Noise to check the effect on our designed system, also channels assumed to be with low correlation coefficient among the channels. In this paper we have used $2 \times 2$ MIMO architecture with low correlation coefficient to study the effect of high SNR on energy efficiency i.e. consumption level of energy per bit at high SNR.

2. Mishra, Chandrakant \& Lal, Sonu, (2016). "Survey Paper on Performance Evaluation of WiMAX (IEEE 802.16) System using Space Time Block Coding Technique", JARCSSE, 6(1), 682-686. In this paper, the review of the MIMO using space time block code on WiMAX (IEEE 802.16) system. The WiMAX (Worldwide Interoperability for Microwaves Access) technology which can offer high speed voice, image, and video and data service up to base on standard 802-16 wireless MAN is configured in the same way as a traditional cellular network. The range of WiMAX makes the system very attractive for users, but there will be slightly higher BER at low SNR.

3. Nidhi Kishor Molgi Suresh R. (2016) “Analysis of MIMO-OFDM using different Modulation Techniques" International Journal of Computer
Applications, Volume 153 - No 6. In this paper authors demonstrated the BER performance of the OFDM system with two different equalizers (ZF and MMSE). Proposed OFDM system is demonstrated using various modulation techniques that is BPSK, QPSK, 16-QAM and 64-QAM using multipath fading channels that is AWGN (Additive White Gaussian Noise) and Rayleigh channel. In this paper, MIMO-OFDM system with an interleaved coded transmission is proposed with different modulation schemes. To demonstrate a coded MIMO-OFDM system is considered with 64 data subcarriers and 16-QAM constellation. Further we also demonstrated system with 128 data subcarriers. To simulate OFDM system Matrix interleaves are used, the cyclic prefix code of length is said to the channel maximum delay, it will prove channel capacity. MIMO-OFDM system increases link reliability, channel capacity and spectral efficiency of multiuser wireless communication.

4. Biswajit, Sahoo \& Ravi Ranjan, Prasad (2015). "BER Analysis of Mobile WiMAX System using LDPC Coding and MIMO System under Rayleigh Channel", International conference on Communication and Signal Processing. In this paper the performance of mobile WiMAX system using LDPC (Low Density Parity Check) coding and MIMO system considering STC techniques under Rayleigh channel is simulated and analyzed. Mobile WiMAX system using LDPC and Turbo coding for different modulation schemes under Rayleigh channel is simulated. From the simulation result, it is concluded that Turbo code gives better BER performance than LDPC code.

Michele, Morelli; Leonardo, Marchetti \& Marco, Moretti, (2014). "Maximum Likelihood Frequency Estimation and Preamble Identification in OFDMA-based WiMAX Systems", IEEE Transactions on Wireless Communications, 13(3), 1582-1592. In this paper investigate the joint maximum likelihood (ML) estimation of the carrier frequency offset (CFO) and preamble index in a multicarrier system compliant with the WiMAX specifications, and derive a novel expression of the relevant CramerRao bound (CRB). Since the exact ML solution is prohibitively complex in its general formulation, suboptimal algorithms are developed which can provide a reasonable trade-off between estimation 
accuracy and processing load. Specifically, show that paper the fractional $\mathrm{CFO}$ can be recovered by combining the ML estimator with an existing algorithm that attains the CRB in all practical scenarios, also discussed OFDM techniques with different parameters.

6. Seyedzadegan, Mojtaba \& Othman, Mohamed, (2013). “IEEE 802.16 WiMAX Overview, WiMAX Architecture", International Journal of Computer Theory and Engineering, 5(5), 784787. This paper was a quick technical overview and covers WiMAX overview of Fundamental Concept; Technology; Standard update and WiMAX architecture like Network and Node Architectures; Physical Layer; MAC Layer). WiMAX is a connection-oriented wide area network. It supports high bandwidth and hundreds of users per channel at speeds similar to currently seen for DSL, Cable or a T1 connection; Promises to provide a range of 30 miles as an alternative to wired broadband like cable and DSL.

\section{WiMAX 802.16 TECHNOLOGIES}

The Communication between embedded systems nowadays is not limited to internal data buses and shared memory but has evolved over the time to include communication channels over wireless protocols as well. The WiMAX (Worldwide Interoperability for Microwave Access) industry forum certifies BWA (Broadband Wireless Access) solutions mainly based on the wireless metropolitan area networking standard developed by IEEE 802.16 group. It is also based on wireless communication that is IP based and is slated on Advanced MIMO technology. Its advances in wireless technologies, such as WiMAX, allow very high data rates, high reliability for communication and through the use of MIMO-OFDM, WiMAX, LTE, Advanced LTE Technologies etc. are part of 4G [1].

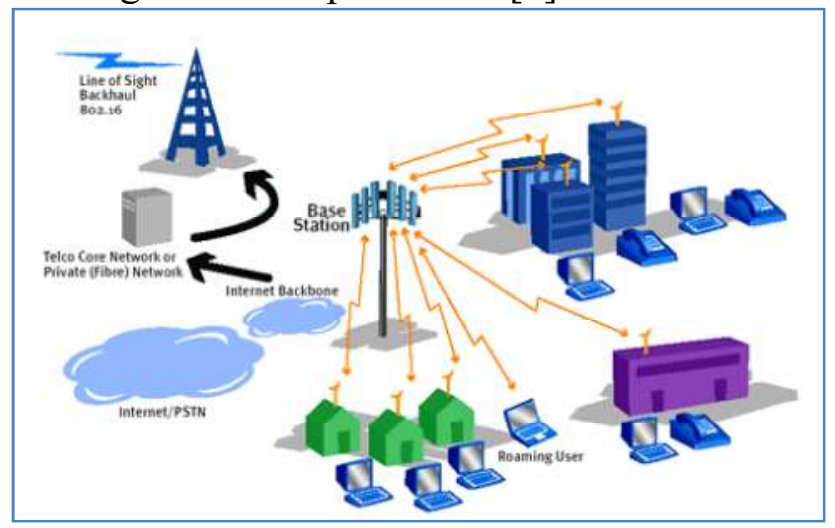

Fig. 2: Architecture of WiMAX
WiMAX technology is expected to meet the needs of a large variety of users from those who are in developed nations wanting to install a new high speed wireless data network very cheaply with the minimum cost and time required. The architecture of WiMAX is show figure 2.

\section{MIMO-OFDM SYSTEM}

Wireless MIMO channels have been recently attracting a great interest since they provide significant improvements in terms of spectral efficiency and reliability with respect to single input single-output (SISO) channels. The gains obtained by the deployment of multiple antennas at both sides of the link are the array gain, the diversity gain, and the multiplexing gain. The array gain is the improvement in signal-to-noise ratio (SNR) obtained by coherently combining the signals on multiple-transmit or multiple-receive dimensions while the diversity gain is the improvement in link reliability obtained by receiving replicas of the information signal through independently fading dimensions.

These gains are not exclusive to MIMO channels and also exist in single-input multiple output (SIMO) and multiple-input single-output (MISO) channels. In contrast, the multiplexing gain, which refers to the increase of rate at no additional power consumption, is a unique characteristic of MIMO channels.

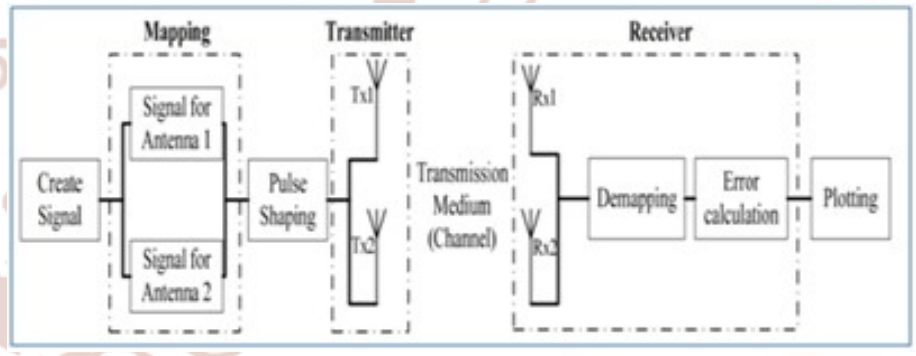

Fig. 3: Simple communication system

\section{COMMUNICATION CHANNEL}

In wireless communication, the data are transmitting through the wireless channel with respective bandwidth to achieve higher data rate and maintain quality of service. The transmitting data has to take environmental challenge when it is on air with against unexpected noise. That's why data has to encounter various effects like multipath delay spread, fading, path loss, Doppler spread and co-channel interference. These environmental effects play the significant role in WiMAX Technology to implement an efficient wireless channels. 
Additive White Gaussian Noise (AWGN),

$>$ Rayleigh Fading Channel,

$>$ Rician Fading channel.

\section{WiMAX Model}

In the Simulation model of the WiMAX, there are some steps for implementation. These are data generation, data randomization, FEC encoder, interleaver, symbol mapper, IFFT modulator at the transmitter side. AWGN channel is used for the WiMAX. At the receiver side FFT modulator, symbol de-mapper, de-interleaver, FEC decoder, Data derandomizer, data collection. OFDM is an important part of the WiMAX.

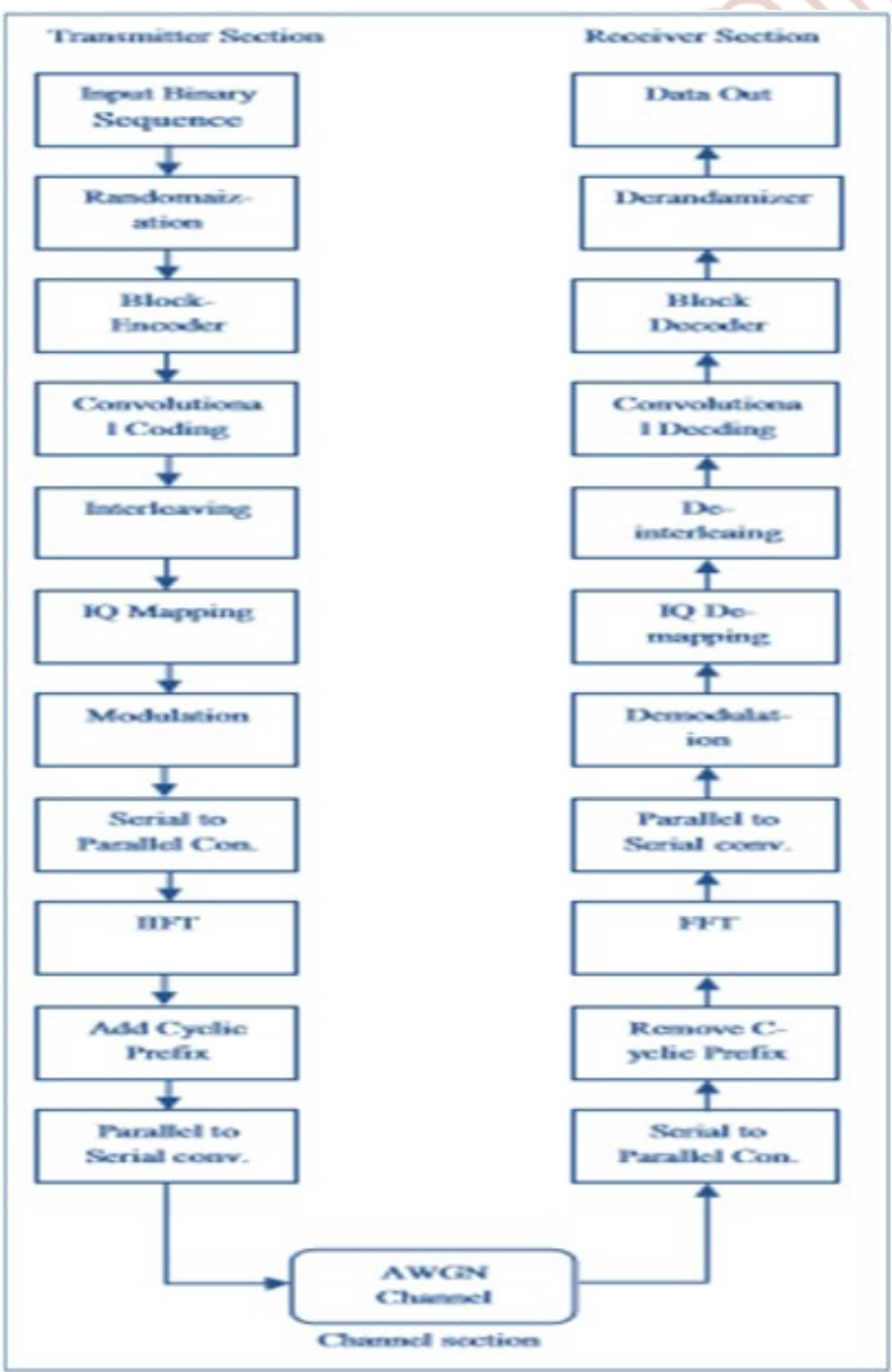

Fig. 4: Simulation Model for WiMAX 802.16e

\section{CONCLUSION}

In this thesis, performance of different MIMO systems is evaluated for different modulation schemes. It is observed that by increasing the order of the MIMO system the capacity of the system increases but along with this the complexity of the overall transceiver increases. Multiple-Input MultipleOutput (MIMO) systems offer considerable increase in data throughput and link range without additional bandwidth or transmit power by using several antennas at transmitter and receiver to improve wireless communication system performance. In this paper review many paper on basses of WiMAX technology.

\section{REFERENCES}

1. Yamini Devlal and M. Awasthi (2015). "Capacity analysis of MIMO technology," IJECS, Vol. 4, Issue 1, pp. 9819-9824.

2. Nimay Ch. Giri, Rupanita Das and SK Mohammed Ali (2014). "BER Analysis and Performance of Mimo-ofdm System Using BPSK Modulation Scheme for Next Generation Communication Systems," IJESRT, Vol. 3, Issue 3, pp. 1622-1629.

3. Mr. Sivanagaraju and Dr. Siddaiah (2014). "Comprehensive Analysis of BER and SNR in OFDM Systems," IJIRCCE, Vol. 2, Issue 2, pp. 3059-3065.

4. Banhijit Bhattacharyya and Somdutta Bhattacharya (2013). "Emerging Fields in 4G Technology, it's Applications \& Beyond-An Overview", International Journal of Information and Computation Technology, 3(4), pp. 251-260.

5. - Shantanu Pathak and Shagun Batra (2012). "Next Generation 4G WiMAX Networks IEEE 802.16 Standard", CoNeCo, WiMo, NLP, CRYPSIS, ICAIT, ICDIP, ITCSE, CS \& IT (7), pp. 507-518.

6. S.M. Lalan Chowdhiury and P. Venkateswaran (2010). "Performance Analysis of WiMAX PHY", IEEE CASCOM Post Graduate Student Paper Conference 2010, Dept of electronics \& TeleCommunication Engg., Jadavpure University, Kollata, India.

7. Jian Li, Guoqing Liu, and Georgios B. Giannakis, (2001). "Carrier Frequency Offset Estimation for OFDM-Based WLANs", IEEE Signal Processing Letters, 8(3).

8. A. F. Sengar, N. Rani, (2005). "Capacity of MIMO Systems with Antenna Selection," IEEE Transaction, Vol. 4, No. 4.

9. Mingji Ban and Sung Ho Cho (2009). “An Efficient Communication Method between Processors in an Embedded Communication 
System", International Conference on New Trends in Information and Service Science, pp. 12681272 .

10. Rehman Talukdar and Mridul Saikia (2014). "Evolution and Innovation in 5G Cellular 12. Communication: A Survey", International Conference on Intelligent Systems and Signal Processing (ISSP), IEEE.

13. M. Divya (2013). "BER performance of BPSK modulation and OFDM-BPSK with Rayleingh multipath channel," IJEAT, vol. 2, Issue 4, pp. 623-626.

14. A. Goldsmith (2003). "Capacity Limits of MIMO Channels," IEEE Communication, Vol. 21, Issue5.

15. Inhye Park, Hyungkeun Lee and Hyukjoon Lee (2013). "Software Optimization for Embedded
Communication System and Beyond: A Study", arXiv: $1407.4335 \mathrm{v} 1$ [cs.NI].

11. Asvin Gohil, Hardik Modi and Shobhit K Patel (2013). "5G Technology of Mobile

Communication System", IEEE, ICOIN 2013, 978-1-4673-5742-5/13.

16. Niraj Kumar Tiwari, Abhishek Kumar Pandey and Prashant Srivastava (2016). "A Study on Next Generation Communication Technology 5G", International Journal of Innovative Research in Computer and Communication Engineering, 4(6).

17. AT\&T 5G LTE field trials start in Q2 (2016). Nokia $5 \mathrm{G} \quad$ LTE Infographic, http://www.fudzilla.com/news/mobile.

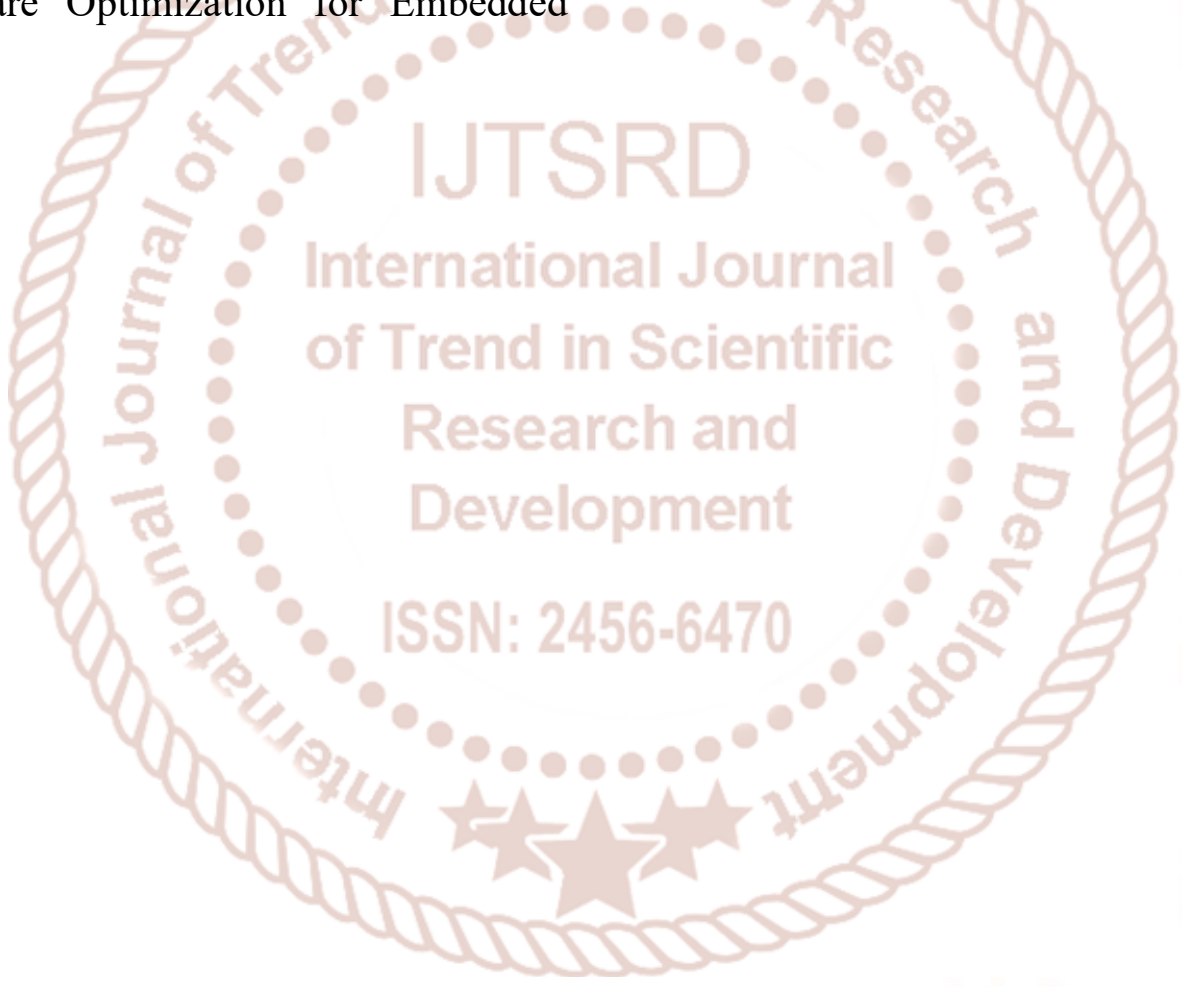

